\title{
Bortezomib induces long term remission in children with immune thrombotic thrombocytopenic purpura, refractory to plasma exchange, glucocorticoids, and rituximab: a report on two cases
}

\author{
Alexei Maschan ${ }^{1}$, Uliana Patrova ${ }^{2}$, Irina Kalinina ${ }^{3}$, Elena Kurnikova ${ }^{3}$, Daria Fedorova ${ }^{4}$, \\ Dina Baidildina ${ }^{2}$, Natalya Kotskaya ${ }^{2}$, Pavel Avdonin ${ }^{5}$, and Galina Novichkova ${ }^{3}$ \\ ${ }^{1}$ Dmitri Rogachev Federal Research Center for Pediatric Hematology, Oncology and \\ Hematology \\ ${ }^{2}$ Dmitri Rogachev National Research Center for Pediatric Hematology, Oncology and \\ Immunology \\ ${ }^{3}$ Dmitri Rogachev National Research Center for Pediatric Hematology, Oncology and \\ Immunology \\ ${ }^{4}$ Dmitry Rogachev National Medical Research Center of Pediatric Hematology Oncology \\ and Immunology \\ ${ }^{5}$ N.K.Koltzov Institute of Developmental Biology
}

October 6, 2020

\begin{abstract}
Acquired thrombotic thrombocytopenic purpura (TTP) in children is a rare but severe disease, which is caused by Immunoglobulin $\mathrm{G}$ antibodies, which inactivate a disintegrin and metalloproteinase with a thrombospondin type 1 motif, member 13 (ADAMTS13). Daily high-volume plasma exchange (PEX) and immunosuppression with glucocorticoids and rituximab is the current standard of treatment for TTP. We report two females aged 5 and 12 years, with TTP, induced by anti-ADAMTS13 inhibitory antibodies who relapsed very shortly after PEX, rituximab and glucocorticoids, in whom long-term remission with disappearance of ADAMTS13 inhibitors was achieved after one course of bortezomib.
\end{abstract}

\section{Introduction}

Acquired thrombotic thrombocytopenic purpura (TTP) in children is a rare but severe disease, which can lead to devastating critical organ damage or even death. ${ }^{1}$ It is caused by Immunoglobulin G (IgG) antibodies, which inactivate a disintegrin and metalloproteinase with a thrombospondin type 1 motif, member 13 (ADAMTS13), which cleaves the ultralarge von Willebrand factor (ULVWF). ${ }^{2}$ In the absence of active ADAMTS13, ULVWF interacts with platelets, inducing the disseminated formation of platelet-rich thrombi both in circulation and in microvessels, which can result in severe ischaemic tissue damage. ${ }^{3}$

Daily high-volume plasma exchange (PEX) and immunosuppression with glucocorticoids and rituximab is the current standard of treatment for TTP, which has decreased mortality rates to less than $10 \%{ }^{4-7}$ Recently, caplacizumab, a nanobody which blocks the A1 domain on ULVWF and prevents its interaction with platelets, has been shown to shorten the interval to remission and also to reduce the likelihood of early death. ${ }^{8}$ However, a significant proportion of patients have TTP refractory to PEX and immunosuppression, or relapse after achieving remission. We describe the cases of two children with immune-mediated TTP, who relapsed shortly after treatment with PEX, glucocorticoids, and rituximab, and subsequently went into continuous complete remission after one course of bortezomib. 


\section{Patient 1}

A 12-year old, girl of Hispanic origin, presented with petechial rash and bruising, jaundice, backaches, nausea, and arthralgia. A complete blood count $(\mathrm{CBC})$ showed thrombocytopenia $\left(11 \mathrm{x} 10^{9} / \mathrm{L}\right)$, anemia $(\mathrm{Hb} ; 9.8 \mathrm{~g} / \mathrm{dl})$, reticulocytosis $(16.9 \%)$, and normal WBC $\left(5.9 \times 10^{9} / \mathrm{L}\right)$. The total bilirubin level was 48 $\mu \mathrm{mol} / \mathrm{L}$, lactate dehydrogenase $(\mathrm{LDH})$ was $514 \mathrm{U} / \mathrm{L}$, and the Coombs test was negative. A diagnosis of immune thrombocytopenia (ITP) was made, and intravenous immunoglobulin (IVIG) $1 \mathrm{~g} / \mathrm{kg}$ body weight (bw) was administered, with no resulting increase in platelets. Subsequently, TTP was suspected, and testing revealed severely decreased activity ADAMTS13, $<5 \%$, which confirmed the diagnosis of TTP and the patient was transferred to our centre. Upon admission, her examination was remarkable for fatigue, cutaneous haemorrhages, and uterine bleeding. The patient's Hb was $5.9 \mathrm{~g} / \mathrm{dl}$, schistocytes were $16 \%$, reticulocytes were $51 \%$, platelets were $16 \times 10^{9} / \mathrm{L}$, and total bilirubin was $54.5 \mu \mathrm{mol} / \mathrm{L}$. Daily PEX was initiated concomitant with methylprednisolone $1000 \mathrm{mg}$ for three days i.v. and rituximab $375 \mathrm{mg} / \mathrm{m}^{2}$ twice weekly for three weeks. After 18 PEXs, the patient's platelet count stabilised above $150 \times 10^{9} / \mathrm{L}$. However, ADAMTS13 was persistently $<5 \%$, and a high level of the ADAMTS13 factor inhibitor persisted. Two days after PEX was electively stopped, the patient's platelet counts dropped again, triggering the resumption of PEX twice weekly, which maintained the platelet count above $300 \times 10^{9} / \mathrm{L}$. Six weeks after the patient's $29^{\text {th }}$ PEX, TTP occurred again, with a drop of platelets to $10 \times 10^{9} /$ L. Thus, because the patient developed TTP refractory to PEX, rituximab, and glucocorticoids, we decided to start therapy with bortezomib, 1.3 $\mathrm{mg} / \mathrm{m}^{2}$ on days $1,4,8$, and 11 . After two additional PEXs and one course of bortezomib the patient's platelet counts, $\mathrm{Hb}$, and LDH all recovered. ADAMTS13 activity reached $81 \%$, and remained within normal limits during 5 years of follow-up. She received one additional course of bortezomib with 21 days interval. There was no toxicity from bortezomib observed, and IgG serum levels were checked several times during follow-up period, during which they never dropped below $7 \mathrm{~g} / \mathrm{L}$, and at the last follow-up was $12.4 \mathrm{~g} / \mathrm{L}$ (figure 1).

\section{Patient 2}

A 5-year old girl was admitted to a hospital with petechiae and ecchymoses after a mild respiratory illness. CBC showed thrombocytopenia $\left(5 \times 10^{9} / \mathrm{L}\right)$, low $\mathrm{Hb}(6.8 \mathrm{~g} / \mathrm{dL})$, and normal WBC $\left(5.5 \times 10^{9} / \mathrm{L}\right)$. The patient was diagnosed with ITP, and IVIG was administered, which resulted in an increased platelet count of $139 \times 10^{9} / \mathrm{L}$ and $\mathrm{Hb}>10.0 \mathrm{~g} / \mathrm{dl}$. A month later the platelets dropped $12 \times 10^{9} / \mathrm{L}$, although her $\mathrm{Hb}$ remained at $12.1 \mathrm{~g} / \mathrm{dL}$, with the presence of rare schistocytes on blood smears. A second course of IVIG was delivered, and her platelet count rose to $301 \times 10^{9} / \mathrm{L}$. After 9 months, the patient developed a petechial rash. Her CBC was indicated thrombocytopenia $\left(19 \times 10^{9} / \mathrm{L}\right)$, low $\mathrm{Hb}(4.9 \mathrm{~g} / \mathrm{dL})$, reticulocytosis $(4.1 \%)$, and the presence of 3-5 schistocytes per high-power view. The patient's LDH was $1183 \mathrm{U} / \mathrm{L}$, urea was 4.5 $\mathrm{mmol} / \mathrm{L}$, and creatinine was $45 \mu \mathrm{mol} / \mathrm{L}$. The direct Coombs test was negative. ADAMTS13 was 0\%, and anti-ADAMTS13 inhibitory antibodies were $>4.5$ Bethesda units. The patient was diagnosed with immune TTP, and high dose IV methylprednisolone $500 \mathrm{mg} / \mathrm{d}$ was administered for 3 days, and FFP infusions of 20 $\mathrm{ml} / \mathrm{kg}$ day were started.

The patient was referred to our centre. Upon admission, the patient was fatigued, with extensive cutaneous haemorrhages. The patient's CBC showed low $\mathrm{Hb}(9.6 \mathrm{~g} / \mathrm{dL})$, reticulocytes (20.5\%), schistocytes (2.8\%), and low platelets $\left(8 \times 10^{9} / \mathrm{L}\right)$. The total bilirubin level was $47.2 \mu \mathrm{mol} / \mathrm{L}, \mathrm{LDH}$ was $1551 \mathrm{U} / \mathrm{L}$, urea was 6.6 $\mathrm{mmol} / \mathrm{L}$, creatinine was $43 \mu \mathrm{mol} / \mathrm{L}$, and proteinuria was indicated at $2.0 \mathrm{~g} / \mathrm{L}$. ADAMTS13 activity was $0 \%$. The patient underwent PEX daily, rituximab $375 \mathrm{mg} / \mathrm{m}^{2}$ was administered twice weekly for 7 doses. After 8 PEX procedures, the patient's platelets recovered to $>150 \times 10^{9} / \mathrm{L}$, and the frequency of the procedure dropped to every other day. Ten days after the last PEX procedure, the patient's platelets dropped to 90 x $10^{9} / \mathrm{L}$, and her $\mathrm{Hb}$ decreased to $10.0 \mathrm{~g} / \mathrm{dL}$. ADAMTS13 activity was $10 \%$. PEX was resumed, and the patient received bortezomib, $1.3 \mathrm{mg} / \mathrm{m}^{2}$ on days $1,4,8$, and 11 . After 2 courses of PEX, the patient's platelets increased to $249 \times 10^{9} / \mathrm{L}$, and ADAMTS13 activity increased to $24 \%$. After two months, her platelets had stabilised at $>300 \times 10^{9} / \mathrm{L}$, ADAMTS13 activity had reached $60 \%$, and inhibitors were no longer detectable. Twelwe months after the last bortezomib dose, the patient remains free of disease, with normal ADAMTS13 activity and no inhibitors (figure 2). 


\section{Discussion}

Treatment of immune-mediated TTP with PEX aims to eliminate ADAMT13 inhibitors from circulation, and to provide ADAMTS13 in amounts sufficient for cleaving ULVWF, while immunosuppression aims to stop further production of inhibitors by B-lymphocytes and plasma cells. It has been shown that treatment with rituximab in addition to PEX and glucocorticoids increases the probability of achieving remission, reduces the probability of relapse, and shortens the interval to remission. ${ }^{6}$ Relapses of TTP are common and are due to the continued production of anti-ADAMTS13 antibodies. Hematologic remission are not equivalent to immunologic remission, as levels of ADAMTS13 activity exceeding 5-10\% are sufficient in a steady state to maintain the appropriate cleavage of ULVWF. ${ }^{9}$ Thus, the ultimate goal of TTP treatment is to restore ADAMTS13 activity to normal by eliminating anti-ADAMTS13 antibodies. Historically, many immunosuppressive interventions, such as vincristine, cyclophosphamide, or splenectomy, have been used in patients with TTP with varying degrees of success.

The proteasome inhibitor bortezomib, have proven to be an effective therapy of multiple myeloma, resulting in the long-term suppression of malignant plasma cells. Bortezomib has been used successfully in the treatment of a variety of autoimmune conditions, including TTP, but only one publication has reported its use in adolescents. ${ }^{10-14}$ In line with published cases, our two patients with short-lived responses to PEX, rituximab, and high-dose glucocorticoids subsequently went into long-term remission with the elimination of inhibitors and normalisation of ADAMTS13 activity after just one course of bortezomib. No side effects of bortezomib were seen, indicating that this treatment is safe and thus suitable for use in children. Monitoring the level of ADAMTS13 activity and inhibitor activity after the achievement of clinical and hematological remission is appropriate for determining the timing of this intervention.

Acknowledgements: Authors wish to thank Vitali Demkin for designing high quality images.

Conflicts of interest : All authors declare no conflict of interest.

\section{Author contribution:}

AM proposed using bortezomib and wrote the text, UP, IK, DF, DB and NK provided clinical care and data retrieval, EK provided plasma exchange, PA assayed ADAMTS13 activity and anti-ADAMTS 13 inhibitors titres, GN participated in discussion and provided valuable advices about paper final version.

\section{References}

1. Joly BS, Stepanian A, Leblanc T, et al. Child-onset and adolescent-onset acquired thrombotic thrombocytopenic purpura with severe ADAMTS13 deficiency: a cohort study of the French national registry for thrombotic microangiopathy. Lancet Haematol . 2016;3(11):e537-e546. doi:10.1016/S23523026(16)30125-9

2. Peyvandi F, Lavoretano S, Palla R, et al. ADAMTS13 and anti-ADAMTS13 antibodies as markers for recurrence of acquired thrombotic thrombocytopenic purpura during remission. Haematologica . 2008;93(2):232-239. doi:10.3324/haematol.11739

3. Furlan M, Robles R, Galbusera M, et al. von Willebrand factor-cleaving protease in thrombotic thrombocytopenic purpura and the hemolytic-uremic syndrome. N Engl J Med. 1998;339(22):1578-1584. doi:10.1056/NEJM199811263392202

4. Tsai HM, Lian EC. Antibodies to von Willebrand factor-cleaving protease in acute thrombotic thrombocytopenic purpura. N Engl J Med. 1998;339(22):1585-1594. doi:10.1056/NEJM199811263392203

5. Rock GA, Shumak KH, Buskard NA, et al. Comparison of plasma exchange with plasma infusion in the treatment of thrombotic thrombocytopenic purpura. Canadian Apheresis Study Group. N Engl J Med. 1991;325(6):393-397. doi:10.1056/NEJM199108083250604

6. Fakhouri F, Vernant JP, Veyradier A, et al. Efficiency of curative and prophylactic treatment with rituximab in ADAMTS13-deficient thrombotic thrombocytopenic purpura: a study of 11 cases. Blood. 2005;106(6):1932-1937. doi:10.1182/blood-2005-03-0848 
7. Jestin M, Benhamou Y, Schelpe AS, et al. Preemptive rituximab prevents long-term relapses in immune-mediated thrombotic thrombocytopenic purpura. Blood . 2018;132(20):2143-2153. doi:10.1182/blood-2018-04-840090

8. Scully M. Rituximab in the treatment of TTP. Hematology. 2012;17 Suppl 1:S22-S24. doi:10.1179/102453312X13336169155178

9. Scully M, Cataland SR, Peyvandi F, et al. Caplacizumab Treatment for Acquired Thrombotic Thrombocytopenic Purpura. N Engl J Med . 2019;380(4):335-346. doi:10.1056/NEJMoa1806311

10. Jin M, Casper TC, Cataland SR, et al. Relationship between ADAMTS13 activity in clinical remission and the risk of TTP relapse. Br J Haematol . 2008;141(5):651-658. doi:10.1111/j.13652141.2008.07107.x

11. Shortt J, Oh DH, Opat SS. ADAMTS13 antibody depletion by bortezomib in thrombotic thrombocytopenic purpura. N Engl J Med. 2013;368(1):90-92.doi:10.1056/NEJMc1213206

12. Patriquin CJ, Thomas MR, Dutt T, et al. Bortezomib in the treatment of refractory thrombotic thrombocytopenic purpura. Br J Haematol. 2016;173(5):779-785. doi:10.1111/bjh.13993

13. Hernández Lorente E, Lluch García R, Monteagudo Santolaya E. Effectiveness of bortezomib in the treatment of thrombotic thrombocytopenic purpura: Case report. Efectividad del bortezomib en el tratamiento de la púrpura trombótica trombocitopénica: descripción de un caso. Med Clin (Barc). 2019;152(6):244-245. doi:10.1016/j.medcli.2018.06.002

14. Mazepa MA, Raval JS, Moll S, Ma A, Park YA. Bortezomib induces clinical remission and reduction of ADAMTS13 inhibitory antibodies in relapsed refractory idiopathic thrombotic thrombocytopenic purpura. Br J Haematol. 2014;164(6):900-902. doi:10.1111/bjh.12696

15. van Balen T, Schreuder MF, de Jong H, van de Kar NC. Refractory thrombotic thrombocytopenic purpura in a 16-year-old girl: successful treatment with bortezomib. Eur J Haematol . 2014;92(1):8082. doi:10.1111/ejh.12206

Figure 1.

Therapies delivered and evolution of laboratory parameters of patient 1 . Solid line indicates platelet counts, dash line depicts ADAMTS13 activity. PEX and arrows indicate plasma exchanges, $\mathrm{R}$ with white arrowheads indicate rituximab, $\mathrm{M}$ followed by circles indicate high-dose methylprednisolone, and $\mathrm{B}$ with black arrowheads indicate bortezomib.

Figure 2 .

Therapies delivered and evolution of laboratory parameters of patient 1. Solid line indicates platelet counts, dash line depicts ADAMTS13 activity. PEX and arrows indicate plasma exchanges, $\mathrm{R}$ with white arrowheads indicate rituximab, $\mathrm{M}$ followed by circles indicate high-dose methylprednisolone, and $\mathrm{B}$ with black arrowheads indicate bortezomib.

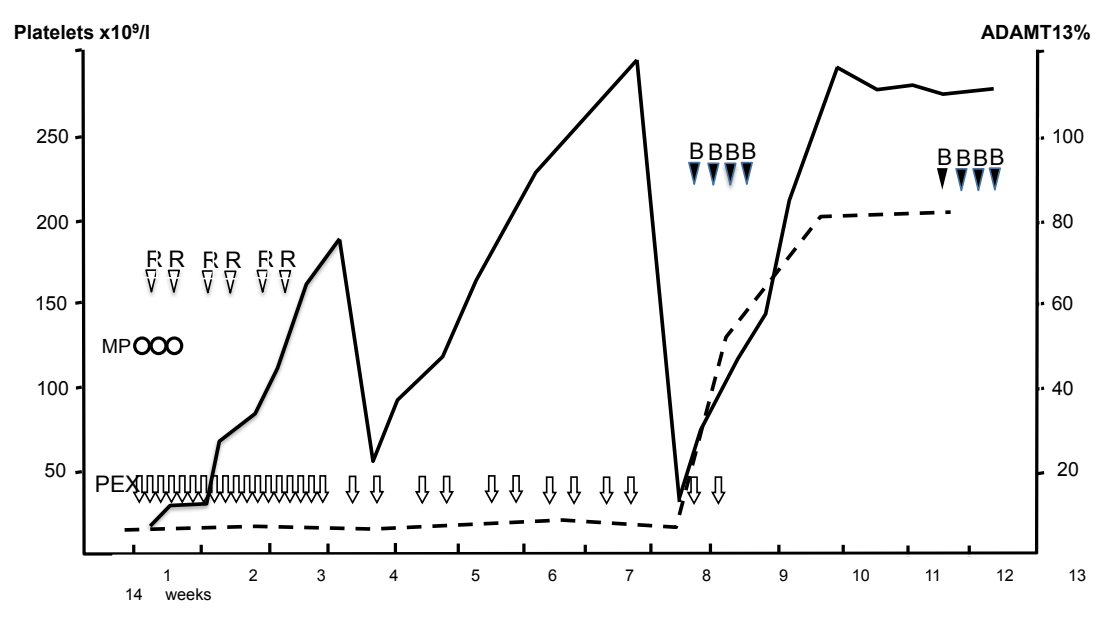




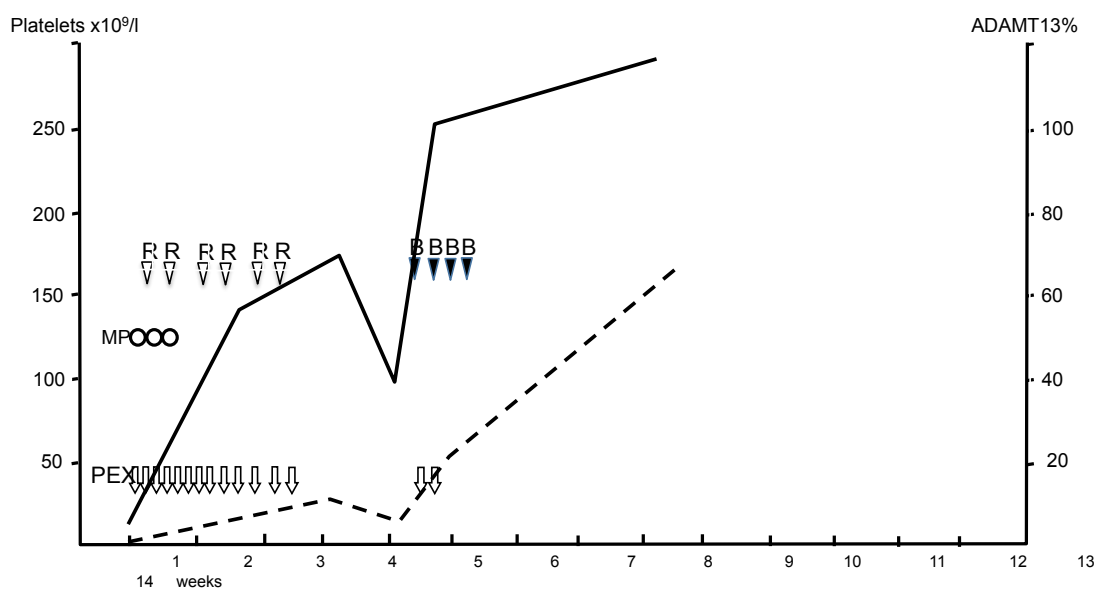

